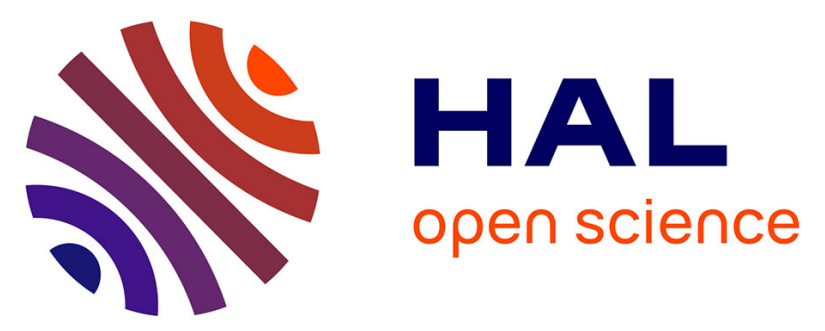

\title{
A new asymmetric division contributes to the continuous production of infective trypanosomes in the tsetse fly.
}

Brice Rotureau, Ines Subota, Johanna Buisson, Philippe Bastin

\section{To cite this version:}

Brice Rotureau, Ines Subota, Johanna Buisson, Philippe Bastin. A new asymmetric division contributes to the continuous production of infective trypanosomes in the tsetse fly.. Development (Cambridge, England), 2012, 139 (10), pp.1842-50. 10.1242/dev.072611 . pasteur-01371317

\section{HAL Id: pasteur-01371317}

\section{https://hal-pasteur.archives-ouvertes.fr/pasteur-01371317}

Submitted on 25 Sep 2016

HAL is a multi-disciplinary open access archive for the deposit and dissemination of scientific research documents, whether they are published or not. The documents may come from teaching and research institutions in France or abroad, or from public or private research centers.
L'archive ouverte pluridisciplinaire HAL, est destinée au dépôt et à la diffusion de documents scientifiques de niveau recherche, publiés ou non, émanant des établissements d'enseignement et de recherche français ou étrangers, des laboratoires publics ou privés.

\section{(ㅇ)(1) $\$$}

Distributed under a Creative Commons Attribution - NonCommerciall 4.0 International 


\title{
A new asymmetric division contributes to the continuous production of infective trypanosomes in the tsetse fly
}

\author{
Brice Rotureau*, Ines Subota, Johanna Buisson and Philippe Bastin
}

\begin{abstract}
SUMMARY
African trypanosomes are flagellated protozoan parasites that cause sleeping sickness and are transmitted by the bite of the tsetse fly. To complete their life cycle in the insect, trypanosomes reach the salivary glands and transform into the metacyclic infective form. The latter are expelled with the saliva at each blood meal during the whole life of the insect. Here, we reveal a means by which the continuous production of infective parasites could be ensured. Dividing trypanosomes present in the salivary glands of infected tsetse flies were monitored by live video-microscopy and by quantitative immunofluorescence analysis using molecular markers for the cytoskeleton and for surface antigens. This revealed the existence of two distinct modes of trypanosome proliferation occurring simultaneously in the salivary glands. The first cycle produces two equivalent cells that are not competent for infection and are attached to the epithelium. This mode of proliferation is predominant at the early steps of infection, ensuring a rapid colonization of the glands. The second mode is more frequent at later stages of infection and involves an asymmetric division. It produces a daughter cell that matures into the infective metacyclic form that is released in the saliva, as demonstrated by the expression of specific molecular markers - the calflagins. The levels of these calcium-binding proteins increase exclusively in the new flagellum during the asymmetric division, showing the commitment of the future daughter cell to differentiation. The coordination of these two alternative cell cycles contributes to the continuous production of infective parasites, turning the tsetse fly into an efficient and long-lasting vector for African trypanosomes.
\end{abstract}

KEY WORDS: Trypanosoma brucei, Glossina, Asymmetric division, Differentiation, Infection

\section{INTRODUCTION}

Trypanosoma brucei are protozoan parasites that are responsible for human African trypanosomiasis or sleeping sickness, a reemerging disease in numerous areas of Africa (Chappuis et al., 2010; Fevre et al., 2008; Simarro et al., 2008). African trypanosomes also cause 'nagana' in cattle with major socioeconomical impacts (Brun et al., 2009). They proliferate as extracellular parasites in the bloodstream of their mammalian host and can, later on, cross the blood-brain barrier to provoke severe neurological symptoms that are fatal in the absence of treatment (Brun et al., 2009). Trypanosomes evade the host immune response by a sophisticated process of antigenic variation involving a dense surface coat of a single type of protein called variant surface glycoprotein (Berriman et al., 2005; Cross, 1975; Taylor and Rudenko, 2006). There is no vaccine and treatments are difficult to administer in rural areas and can provoke severe side effects (Brun et al., 2009).

African trypanosomes are transmitted by the bite of the tsetse fly and the geographical repartition of Glossina vectors governs the distribution of sleeping sickness (Brun et al., 2009; Simarro et al., 2008). A tsetse fly becomes infected during a blood meal on an infected mammal. To complete their life cycle, trypanosomes need to reach the tsetse salivary glands and to transform into infective metacyclic parasites that are found free in the saliva. This is not direct and requires several migration, proliferation and differentiation steps that take place in a strictly defined

Trypanosome Cell Biology Unit, Institut Pasteur \& CNRS, URA 2581, 25 rue du Docteur Roux, 75015 Paris, France.

*Author for correspondence (rotureau@pasteur.fr)

Accepted 30 January 2012 chronological order in specific fly tissues (up to 3 weeks) (Oberle et al., 2010; Rotureau et al., 2011; Sharma et al., 2008; Van Den Abbeele et al., 1999; Vickerman, 1985). Once trypanosomes are present in the salivary glands, a fly can produce hundreds of metacyclic parasites per day (Otieno and Darji, 1979) and remains infective for its whole life ( $\sim 3$ months), a meaningful fact considering that a rather low proportion of flies is infected $(<0.1 \%)$ (Aksoy et al., 2003; Brun et al., 2009). Moreover, fly behavior is altered by trypanosome infection that modifies the salivary composition, resulting in a drastically reduced anti-hemostatic potential and a hampered feeding performance. This forces infected flies to feed more often and for longer periods, which favors parasite transmission (Van Den Abbeele et al., 2010). Despite its crucial role in transmission, the way in which the continuous production of infective parasites is ensured in the salivary glands of the tsetse remains undetermined.

At least three main parasite morphotypes have been reported in the tsetse salivary glands (supplementary material Table S1): one epimastigote stage, where the basal body of the flagellum, which is linked to the DNA of the single mitochondrion (kinetoplast) (Robinson and Gull, 1991), is found in an anterior position relative to the nucleus; and two trypomastigote stages, where the basal body/kinetoplast complex is present at the posterior end of the cell. The epimastigote form and the first type of trypomastigote, called pre-metacyclic, are both attached to the salivary gland epithelium via elaborate extensions of their flagellum membrane (Tetley and Vickerman, 1985). The second type of trypomastigote is the freeswimming metacyclic form, the only one to be infective for mammals. Dividing attached epimastigotes have been reported (Steiger, 1973; Tetley and Vickerman, 1985; Vickerman, 1985; Vickerman et al., 1988) but their abundance, their mode of division and their significance for the parasite cycle has not been established. Some of these epimastigotes were recently observed to 
complete the first meiotic division (Peacock et al., 2011); however, the second step of the meiotic program, the subsequent cell fusion and the significance of these phenomena in trypanosome development remain to be clarified. Metacyclic trypanosomes express a variant surface glycoprotein coat (Vickerman, 1978) and are expelled at each blood meal with the saliva. They have been proposed to derive from the progressive migration of the kinetoplast to the posterior end, accompanied by the resorption of the flagellum membrane and the detachment from the epithelium, concomitant with the acquisition of a variant surface glycoprotein coat (Tetley and Vickerman, 1985). However, this model might not completely explain the continuous production of infective metacyclic parasites throughout the whole life of the vector (Sharma et al., 2009). We therefore investigated how trypanosomes proliferate in the salivary glands and how this process could be coupled to differentiation.

Trypanosomes were monitored by live video microscopy and by immunofluorescence using different molecular probes for cytoskeleton components, cell cycle markers and surface antigens to examine cell morphogenesis and parasite division in the salivary glands. We reveal the existence of two alternative modes of trypanosome proliferation occurring simultaneously in the salivary glands. The first one produces two equivalent progenies that are not competent for infection and remain attached to the epithelium. The second one results in an asymmetric division, producing a daughter that is apparently similar to its mother, and a daughter that differentiates to become infective. Remarkably, the first mode of proliferation is abundant at the early stages of infection, ensuring colonization of the salivary glands, whereas the second one is predominant at later stages. The balance between these two modes of division contributes to the continuous production of infective parasites in the saliva to ensure efficient transmission to the next mammalian host.

\section{MATERIALS AND METHODS}

\section{Trypanosome strains and cultures}

The pleomorphic strain Trypanosoma brucei brucei AnTat1.1 (Le Ray et al., 1977) was used throughout this study. AnTat1.1 procyclic trypanosomes nucleofected with the GFP-expressing construct pHD67E (Bingle et al., 2001) were also used to monitor parasite attachment and development within tissues. Cultures of bloodstream and procyclic forms were performed as described previously (Rotureau et al., 2011).

\section{Tsetse fly infection, maintenance and dissection}

A total of 2530 teneral males of Glossina morsitans morsitans from 8 to 96 hours post-eclosion were obtained from the UMR 177 IRD-CIRAD, Campus International de Baillarguet, Montpellier, France. Tsetse flies were infected, maintained and dissected as described previously (Rotureau et al., 2011). Flies were starved for at least 48 hours before being dissected 15 to 55 days post-ingestion. Tissues were then directly observed under the microscope or rapidly opened and flushed to resuspend parasites in culture medium or phosphate-buffered saline for further experiments.

\section{Immunofluorescence}

Cells were treated for immunofluorescence after paraformaldehyde or methanol fixation as described previously (Rotureau et al., 2011). DNA was stained with 4',6-diamidino-2-phenylindole (DAPI). The mAb25 antibody (IgG2a) recognizes a protein found all along the axoneme (Pradel et al., 2006), whereas the mAb22 (IgM) antibody detects an as yet unidentified antigen found at the proximal zone of both the mature and the pro-basal body (Bonhivers et al., 2008). The L8C4 antibody (IgG1) targets an epitope of the PFR2 protein (Kohl et al., 1999). The YL1/2 (Kilmartin et al., 1982) and KMX1 (Birkett et al., 1985) antibodies recognize tyrosinated $\alpha$-tubulin and $\beta$-tubulin, respectively. The anti-CRD (Zamze et al., 1988) and the anti-BARP (Urwyler et al., 2007) antibodies were, respectively, used to label the cross-reactive determinant of the glycosylphosphatidylinositol anchors of the variant surface glycoproteins and the BARP antigens after a 30 -second methanol fixation protocol. Parasites from dissected organs were immediately air-dried, fixed in a methanol bath at $-20^{\circ} \mathrm{C}$ for about 30 seconds and re-hydrated in phosphate-buffered saline for 10 minutes before immunostaining. A calflagin antiserum that labels all proteins of the calflagin family was used after a 30-second methanol fixation (Giroud et al., 2009). The anti-SCC1 antibody targets the sister chromatid cohesin component 1 found in the nucleus only during the $\mathrm{S}$ and G2 phases of the cell cycle (Sharma et al., 2008). For each antibody, immunofluorescence experiments were repeated on trypanosomes issued from three to 13 different flies and from at least three distinct experimental infections. Phalloidin coupled to Alexa Fluor 555 (Invitrogen) was used according to the manufacturer's recommendations to label actin fibers in whole fly tissues after overnight paraformaldehyde fixation.

\section{Measurements and analyses}

Samples were observed either with: (1) a DMR microscope (Leica) and images were captured with a CoolSnap HQ camera (Roper Scientific); (2) with a DMI4000 microscope (Leica) and images were acquired with a Retiga-SRV camera (Q-Imaging); or (3) with a digital D-Eclipse EZC1si confocal system (Nikon) installed on an Eclipse TE2000-E inverted microscope (Nikon). Pictures were analyzed and cell parameters were measured using the IPLab Spectrum 3.9 software (Scanalytics \& BD Biosciences), the ImageJ $1.38 \times$ software $(\mathrm{NIH})$ or the NIS-elements software (Nikon). For clarity purposes, the brightness and contrast of several pictures were adjusted after their analysis in accordance with editorial policies. To compare fluorescence intensities, background fluorescence was removed from all pictures and values were normalized to the highest value of each batch of experiment (Figs 3, 4). Measured parameters are: the total length of the cell (Total); the distance between the center of the most anterior nucleus and the anterior end of the cell (Nucleus 1/N1-Anterior); the distance between the center of the most anterior nucleus and the posterior end of the cell (Nucleus 1/N1-Posterior); the distance between the center of the most anterior nucleus and the kinetoplast associated with the old flagellum (Nucleus 1/N1-Old kinetoplast/K1); the distance between the kinetoplast associated with the old flagellum and the posterior end of the cell (Old kinetoplast/K1-Posterior); the length of the old flagellum measured on phase-contrast pictures (Old flagellum); the length of the axoneme of the old flagellum measured on pictures of mAb25 stained cells [Old flagellum (mAb25)]; the distance between the center of the most anterior nucleus and the kinetoplast associated with the new flagellum (Nucleus 1/N1-New kinetoplast/K2); the distance between the two kinetoplasts (Old kinetoplast/K1-New kinetoplast/K2); the distance between the kinetoplast associated with the new flagellum and the posterior end of the cell (New kinetoplast/K2-Posterior); the length of the new flagellum measured on phase-contrast pictures (New flagellum); the length of the axoneme of the new flagellum measured on pictures of mAb25 stained cells [New flagellum (mAb25)]; the distance between the center of the two nuclei (Nucleus 1/N1-Nucleus 2/N2); the distance between the center of the most posterior nucleus and the anterior end of the cell (Nucleus 2 / N2-Anterior); the distance between the center of the most posterior nucleus and the posterior end of the cell (Nucleus 2/N2-Posterior); and the distance between the center of the most posterior nucleus and the kinetoplast associated with the new flagellum (Nucleus 2/N2-New kinetoplast/K2). Statistical analyses were performed in Excel or with the KaleidaGraph V.4.0 software (Synergy Software). Normalized fluorescence intensity measurements (arbitrary units), morphometric measurements $(\mu \mathrm{m})$ and cell counts were carried out as previously described (Rotureau et al., 2011) and plotted as mean \pm s.d. Two-tailed unpaired $t$-tests or one-way ANOVA tests, with intergroup comparisons by Tukey ad-hoc post-tests with $\alpha=0.05$, were performed, and significant results were indicated with $* * * P<0.0001, * * P<0.001$ and $* P<0.01$ (Fig. 1L, Fig. 2L, Fig. 3K, Fig. 4E,H, Fig. 5A). 

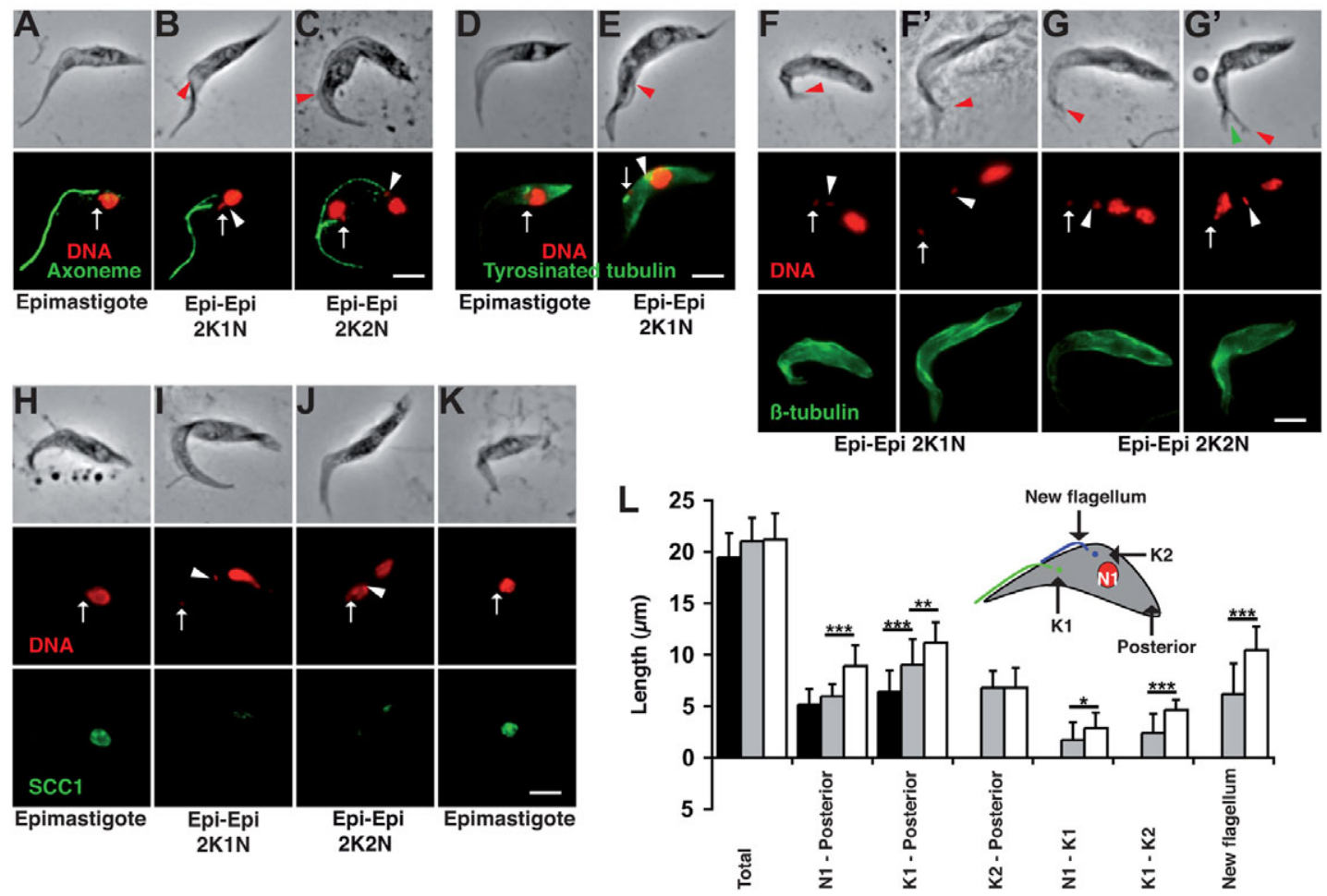

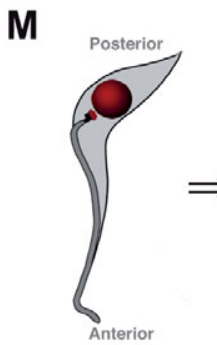

Epimastigote

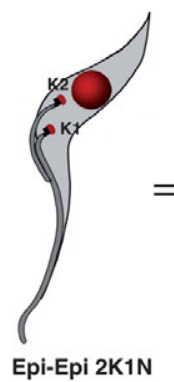

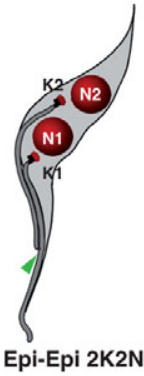

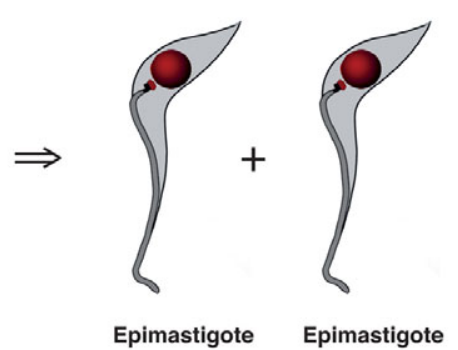

Fig. 1. The 'epi-epi' cell cycle produces two similar epimastigote daughter cells. (A-K) Trypanosomes from salivary glands were fixed in methanol and stained with 4',6-diamidino-2-phenylindole (DNA in red in A-K) and mAb25 (axoneme in green in A-C), YL1/2 (tyrosinated $\alpha$-tubulin in green in $\mathrm{D}, \mathrm{E}), \mathrm{KMX} 1$ ( $\beta$-tubulin in green in $\mathrm{F}-\mathrm{G}^{\prime}$ ) or anti-SCC1 (sister chromatid cohesin component 1 in green in $\mathrm{H}-\mathrm{K}$ ). $1 \mathrm{~K} 1 \mathrm{~N}$ epimastigotes $(A, D, H, K), 2 K 1 N$ 'epi-epi' cells $\left(B, E-F^{\prime}, I\right)$ and $2 K 2 N$ 'epi-epi' cells $\left(C, G-G{ }^{\prime}, J\right)$ were identified according to the number and relative positioning of their kinetoplasts and nuclei. The positions of the old (white arrow) and new (white arrowhead) kinetoplasts are indicated, as well as the tip of the new flagellum (red arrowhead) when present. Scale bars: $5 \mu \mathrm{m}$. (L) Mean values \pm s.d. (in $\mu \mathrm{m}$ ) of various morphometric parameters for $1 \mathrm{~K} 1 \mathrm{~N}$ (black, $n=38), 2 \mathrm{~K} 1 \mathrm{~N}$ (gray, $n=42$ ) and $2 \mathrm{~K} 2 \mathrm{~N}$ (white, $n=31$ ) trypanosomes from salivary glands. Measured parameters are the total cell length, the distance between the nucleus 1 (N1) and the posterior end, the distance between the old kinetoplast (K1) and the posterior end, the distance between the new kinetoplast (K2) and the posterior end, the distance between the nucleus 1 (N1) and the old kinetoplast (K1), the distance between the two kinetoplasts (K1-K2) and the length of the new flagellum. ${ }^{* *} P<0.0001,{ }^{*} P<0.001$ or ${ }^{*} P<0.01$, intergroup comparisons from one-way ANOVA. (M) Schematics of the 'epi-epi' cell cycle. Cytokinesis initiates at the tip of the new flagellum and follows the path of the flagellum (green arrowhead). N1 and N2, nuclei 1 and 2; K1 and K2, kinetoplasts associated with the old and new flagella, respectively.

\section{RESULTS}

\section{Two modes of trypanosome proliferation in the salivary glands}

In order to unravel the development of trypanosomes in the tsetse salivary glands, a total of 2530 teneral males of Glossina morsitans morsitans were infected with the pleomorphic strain Trypanosoma brucei brucei AnTat1.1 in 35 separate experiments. Out of the 594 flies dissected 15 to 55 days post-ingestion, $14.5 \%$ presented a salivary gland infection. This rate of mature infections was comparable with those previously observed (Peacock et al., 2007; Peacock et al., 2011; Rotureau et al., 2011; Sharma et al., 2008; Van Den Abbeele et al., 1999; Vassella et al., 2009). Epimastigotes attached to the salivary glands were first examined directly in vivo (supplementary material Movie 1). Parasites expressing a cytosolic GFP were also used to verify the attachment of dividing cells in fixed salivary glands stained with phalloidin, a marker for the actin network of epithelial cells (supplementary material Movie 2 and Fig. S1). Details were obtained by monitoring live dividing epimastigotes released from opened salivary glands (supplementary material Movie 3), and by staining the flagellar axoneme (Fig. 1AC, Fig. 2A,B) and paraflagellar rod (PFR in supplementary material Fig. S2), $\beta$-tubulin in the microtubule corset (Fig. 1F-G', Fig. 2E$\mathrm{F}^{\prime}$ ) and tyrosinated $\alpha$-tubulin (Fig. 1D,E, Fig. 2C,D) as a marker of cytoskeleton elongation (Sherwin and Gull, 1989). The cell cycle status was verified by labeling the DNA with 4',6-diamidino2-phenylindole (DAPI staining in red with $\mathrm{N}$ for nucleus and $\mathrm{K}$ for 
kinetoplast) and the cohesin sub-unit SCC1 (Fig. 1H-K, Fig. 2G$\mathrm{K}$ ), found exclusively in the nucleus during S and G2 phases but not during mitosis (Sharma et al., 2008).

In salivary gland epimastigotes, we observed that cell proliferation starts with the duplication of the basal bodies/kinetoplast complex and the assembly of the new flagellum (Fig. 1B, Fig. 2A; supplementary material Fig. S2B,E). However, the subsequent migration of the kinetoplast associated with the new flagellum (K2) shows two different patterns: it occurs towards the anterior end of the cell in the first case (Fig. 1B,L; supplementary material Fig. S2B) and towards the posterior end of the cell, on the other side of the nucleus, in the second case (Fig. 2A,L; supplementary material Fig. S2E). These two types of kinetoplast positioning are still observed after nuclear mitosis in dividing cells with two kinetoplasts and two nuclei $(2 \mathrm{~K} 2 \mathrm{~N}$ cells in Fig. 1C,G$\mathrm{G}^{\prime}, \mathrm{J}$, Fig. 2F-F', I; supplementary material Fig. S2C,F). For clarity, we have chosen to describe these two profiles separately (Figs 1, 2).

In the first case, the posterior end of the epimastigote cell elongates, as shown by the incorporation of tyrosinated tubulin (Fig. 1D). The new flagellum elongates to reach $\sim 6 \mu \mathrm{m}$ (Fig. 1L) with its tip attached to the old flagellum in $79 \%$ of the cells with two kinetoplasts and one nucleus $(n=42)$. The distance between the two kinetoplasts (K1-K2), both anterior to the nucleus, increases concomitant to the posterior end elongation (Fig. 1L). As shown by the signal of the cohesin marker in $2 \mathrm{~K} 1 \mathrm{~N}$ cells (Fig. 1I), the nucleus completes mitosis after kinetoplast duplication, resulting in a cell with two kinetoplasts and two nuclei ( $2 \mathrm{~K} 2 \mathrm{~N}$ in Fig. 1C,G$\mathrm{G}^{\prime}, \mathrm{J}$; supplementary material Fig. S2C). The new flagellum continues its growth to reach $\sim 10 \mu \mathrm{m}$ (Fig. 1L), still being attached to the old flagellum by its tip in $65 \%$ of the $2 \mathrm{~K} 2 \mathrm{~N}$ cells $(n=31)$. After mitosis, nuclei segregate extensively in such a way that, prior to cell division, the most anterior nucleus (N1) is positioned between the two kinetoplasts (Fig. $1 \mathrm{C}, \mathrm{G}-\mathrm{G}^{\prime}, \mathrm{J}$; supplementary material Fig. S2C), resulting in an organizational scheme along the antero-posterior axis summarized as 'K1-N1-K2-N2' (Fig. 1M). This profile can be compared with the procyclic cell cycle occurring in the midgut, except that the kinetoplasts are found in an anterior position relative to the nuclei. Cytokinesis then initiates close to the tip of the new flagellum after disconnection from the old one (Fig. 1G'; supplementary material Fig. S2C), producing two equivalent daughter cells with an epimastigote configuration (Fig. 1M). Hence, the name 'epi-epi' division is proposed for this mode of cell proliferation.

Surprisingly, we also detected numerous individuals that did not match the 'epi-epi' configuration: these trypanosomes possess two kinetoplasts, two flagella and one or two nuclei, but the position of the new basal body is posterior to the nucleus (Fig. 2A,C,E-E',H; supplementary material Fig. S2E). Incorporation of tyrosinated tubulin mostly takes place at the posterior end (Fig. 2C), whereas the new flagellum elongates to reach $\sim 10 \mu \mathrm{m}$ before mitosis and $\sim 13 \mu \mathrm{m}$ before the end of cell division (Fig. 2L). In contrast to the 'epi-epi' division, the tip of the new flagellum is frequently detached from the old flagellum: only $49 \%(n=37)$ and $36 \%(n=22)$ of the cells with one and two nuclei, respectively, showed an apparent connection between the tip of the new flagellum and the side of the old one (Fig. 2F-F'). The distribution of the data presented in supplementary material Table $\mathrm{S} 2$ provides information about the numerous intermediate situations reflecting the subcellular events occurring during this division. The 57 asymmetrically dividing cells in which the distances between the kinetoplast associated with the new flagellum (K2) and the posterior end of the cell (K2-Posterior) have been measured were observed at different steps of the division process (compare range and standard deviation of these two parameters). During the $2 \mathrm{~K} 1 \mathrm{~N}$ to $2 \mathrm{~K} 2 \mathrm{~N}$ transition, the distance between the new kinetoplast (K2) and the posterior end decreases from $5 \mu \mathrm{m}$ to $3.8 \mu \mathrm{m}$, and the distance between the anterior nucleus (N1) and the new kinetoplast (K2) (N1-K2) significantly increases from $2 \mu \mathrm{m}$ to $3.8 \mu \mathrm{m}$; the distance between the anterior nucleus $(\mathrm{N} 1)$ and the posterior end (N1-Posterior), however, remains broadly constant at 7-8 $\mu \mathrm{m}$, as does the total cell length at $20 \mu \mathrm{m}$ (supplementary material Table S2; Fig. 1L, Fig. 2L). Thus, it appears that one major event of this division is the early migration of the kinetoplast associated with the new flagellum to the posterior side of the nucleus. After this repositioning of the kinetoplast associated with the new flagellum, mitosis occurs lateral to the antero-posterior axis and the two nuclei remain relatively close to each other (Fig. 2F- $\mathrm{F}^{\prime}$,I, supplementary material Fig. S2F), resulting in a novel organizational scheme along the antero-posterior axis of the cell that is 'K1-N1-N2-K2' (Fig. 2M). Cytokinesis begins along the axis of the new flagellum before the completion of mitosis (Fig. 2A,F-F'). At this stage, the total cell length, the inter-kinetoplast distance and the position of each kinetoplast relative to the posterior and the anterior end of the cell are comparable with the parameters measured for the 'epi-epi' mode of proliferation (supplementary material Table S2; Fig. 1L, Fig. 2L). These morphometric data indicate that mitosis is delayed in these cells compared with 'epi-epi' dividing parasites. This asymmetric division results in two distinct daughter cells: the one inheriting the old flagellum displays the epimastigote configuration and the one inheriting the new flagellum adopts the trypomastigote configuration (Fig. 2M). Hence, the term 'epi-trypo' division is proposed.

\section{Molecular markers for differentiation}

The trypomastigote daughter cell resulting from the asymmetric division could be the precursor of the infective metacyclic form. Such an intermediate form has been called pre-metacyclic and shown to remain attached to the epithelium (Steiger, 1973; Tetley et al., 1987; Tetley and Vickerman, 1985). The maturation of the pre-metacyclic into the metacyclic form involves morphological changes such as rounding-up of the posterior end, elongation of the flagellum and repositioning of the kinetoplast to the far posterior end (Fig. 2J,K, Fig. 3D,E,I,J, Fig. 4C,D; supplementary material $\mathrm{S} 2 \mathrm{G}, \mathrm{H})$. It is noteworthy that the posterior migration of the kinetoplast associated with the new flagellum initiated during the asymmetric 'epi-trypo' division seems to go further on during the maturation of the $1 \mathrm{~K} 1 \mathrm{~N}$ trypomastigote daughter cell with a broadly linear distribution $\left(\mathrm{R}^{2}=0.64\right)$ of the distance between the kinetoplast and the posterior end in the range of 6.9 to $0.1 \mu \mathrm{m}$ (Kinetoplast-Posterior in supplementary material Table S2).

To assess whether the trypomastigote daughter cell issued from the 'epi-trypo' division is the precursor of the infective metacyclic form, we looked for molecular markers expressed in the premetacyclic and metacyclic stages that could appear as early as during the asymmetric 'epi-trypo' division. The transition from the epimastigote to the trypomastigote metacyclic form is accompanied by the disappearance of the epimastigote-specific surface antigen BARP (Urwyler et al., 2007) and the acquisition of a variant surface glycoprotein coat (Tetley et al., 1987; Tetley and Vickerman, 1985). Immunofluorescence analyses with an antiBARP antiserum revealed that attached epimastigotes (Fig. 3A) as well as 'epi-epi' dividing cells (Fig. 3B) were strongly positive for BARP, whereas metacyclic parasites turned out to be negative, as 

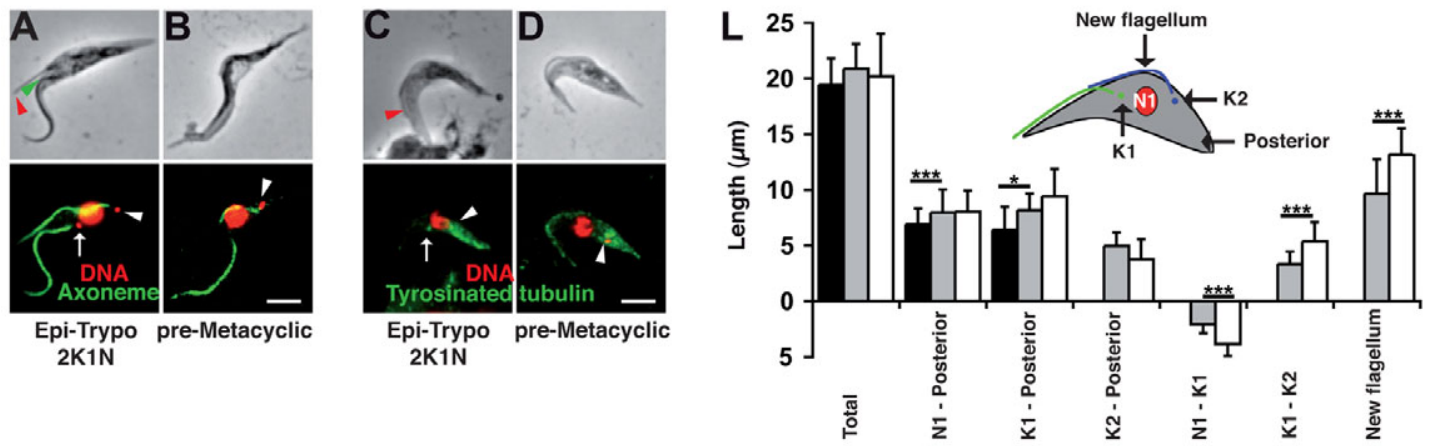

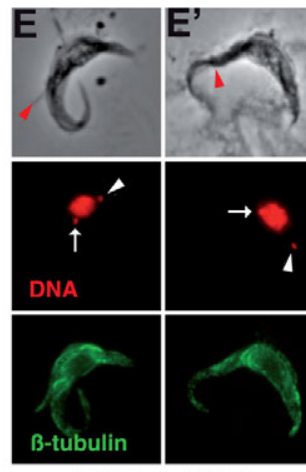

Epi-Trypo 2K1N

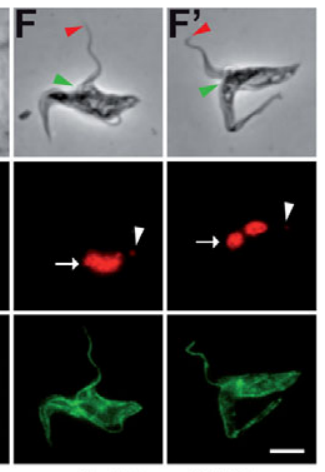

Epi-Trypo 2K2N

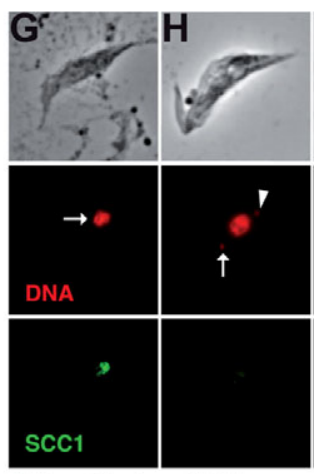

Epimastigote Epi-Trypo

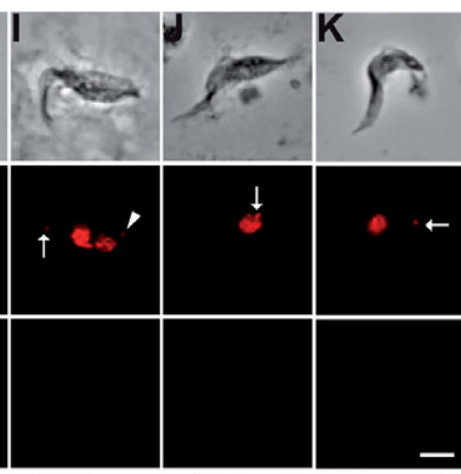

Epi-Trypo pre-Metacyclic Metacyclic $2 \mathrm{~K} 2 \mathrm{~N}$

M

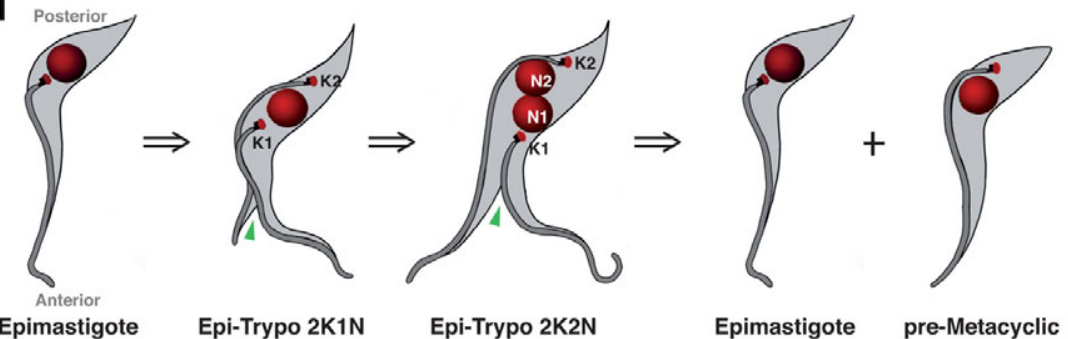

Fig. 2. The 'epi-trypo' cell cycle results in an asymmetric division that produces one epimastigote and one trypomastigote cell.

(A-K) Trypanosomes from salivary glands were fixed in methanol and stained with 4',6-diamidino-2-phenylindole (DNA in red in A-K) and mAb25 (axoneme in green in $A, B), Y L 1 / 2$ (tyrosinated $\alpha$-tubulin in green in C,D), KMX1 ( $\beta$-tubulin in green in E,F) or anti-SCC1 (sister chromatid cohesin component 1 in green in G-K). 1K1N epimastigotes (G), 2K1N 'epi-trypo' cells (A,C,E,E',H), 2K2N 'epi-trypo' cells (F, $\left.F^{\prime}, I\right)$, pre-metacyclic cells (B,D,J) and metacyclic trypomastigotes $(K)$ were identified according to the number and relative positioning of their kinetoplasts and nuclei. The positions of the old (white arrow) and new (white arrowhead) kinetoplasts are indicated, as well as the tip of the new flagellum (red arrowhead) when present. Scale bars: $5 \mu \mathrm{m}$. (L) Mean values \pm s.d. (in $\mu \mathrm{m}$ ) of various morphometric parameters for $1 \mathrm{~K} 1 \mathrm{~N}$ (black, $n=38$ ), $2 \mathrm{~K} 1 \mathrm{~N}(\mathrm{gray}, n=37)$ and $2 \mathrm{~K} 2 \mathrm{~N}$ (white, $n=22$ ) trypanosomes from salivary glands. Measured parameters are the total cell length, the distance between the nucleus 1 (N1) and the posterior end, the distance between the old kinetoplast (K1) and the posterior end, the distance between the new kinetoplast (K2) and the posterior end, the distance between the nucleus 1 (N1) and the old kinetoplast (K1), the distance between the two kinetoplasts (K1-K2) and the length of the new flagellum. ${ }^{*} * P<0.0001$ or ${ }^{*} P<0.01$, intergroup comparisons from one-way ANOVA. (M) Schematics of the 'epi-trypo' cell cycle. Cytokinesis initiates at the tip of the new flagellum and follows the path of the flagellum (green arrowhead). N1 and N2, nuclei 1 and 2; K1 and $\mathrm{K} 2$, kinetoplasts associated with the old and new flagella, respectively.

expected (Fig. 3E). A decrease in the abundance of the BARP antigen was observed in pre-metacyclic cells (Fig. 3D) but not in asymmetrically dividing 'epi-trypo' cells (Fig. 3C).

Monitoring variant surface glycoproteins is more difficult as only a single type of variant surface glycoproteins is expressed at a time, but it can be the product of any single gene from the metacyclic repertoire, and the encoded proteins are extremely divergent (Berriman et al., 2005). However, the variant surface glycoproteins share a conserved region called the cross-reacting determinant (CRD) that is implicated in glycosyl-phosphatidylinositol anchoring to the membrane. This cross-reacting determinant is buried in the variant surface glycoprotein molecules and only accessible to antibodies upon phospholipase treatment (Cardoso de Almeida and Turner, 1983; Grab et al., 1984; Zamze et al., 1988). We reasoned that this domain of the glycosyl-phosphatidylinositol anchor of the variant surface glycoprotein could be exposed upon methanol fixation, and this exposure would offer the possibility of probing for the presence of a variant surface glycoprotein coat by immunofluorescence regardless of the type expressed. After a 30second fixation in methanol, the anti-CRD polyclonal antibody was incubated with a mixture of cells containing $50 \%$ of cultured bloodstream and $50 \%$ of cultured procyclic trypanosomes. A strong fluorescent signal at the surface of bloodstream cells was observed, whereas procyclic parasites were all negative, proving the efficacy 

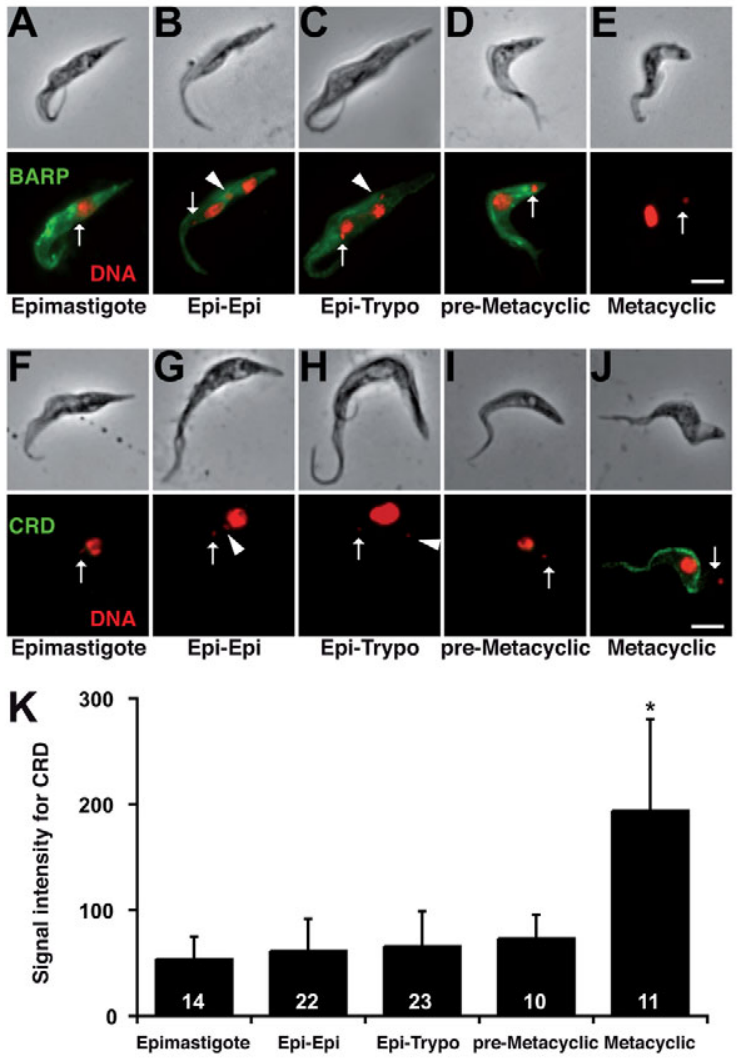

Fig. 3. Surface coat replacement in pre-metacyclic parasites.

(A-J) Trypanosomes from salivary glands were fixed in methanol for 30 seconds and stained with 4',6-diamidino-2-phenylindole (DNA in red in A-J) and anti-BARP (BARP antigen coat in green in A-E) or anti-CRD (cross-reactive determinant of the variant surface glycoprotein glycosylphosphatidylinositol anchor in green in F-J). Epimastigotes (A,F), 'epiepi' cells $(B, G)$, 'epi-trypo' cells $(C, H)$, pre-metacyclic cells $(D, I)$ and metacyclic trypomastigotes $(E, J)$ were identified according to the number and relative positioning of their kinetoplasts and nuclei. The positions of the old (white arrow) and new (white arrowhead) kinetoplasts are indicated. Scale bars: $5 \mu \mathrm{m}$. (K) Mean normalized fluorescence intensity \pm s.d. (in arbitrary units) obtained by staining the cross-reactive determinant (CRD) of the variant surface glycoprotein glycosyl-phosphatidylinositol anchor of the indicated trypanosome stages from salivary glands. $n$ is given inside columns; ${ }^{*} P<0.001$, twotailed unpaired Student's $t$-test when compared with all other groups.

of the assay (supplementary material Fig. S3A). The anti-CRD antibody was then used under the same conditions to label parasites issued from the salivary glands (Fig. 3F-K; supplementary material Fig. S3B). As expected, the epimastigote cells appeared negative (Fig. 3F,K; supplementary material Fig. S3B), whereas the metacyclic parasites were positive (Fig. 3J,K; supplementary material Fig. S3B) with a bright staining at their surface. However, neither the 'epi-epi' (Fig. 3G,K) nor the 'epi-trypo' dividing cells (Fig. 3H,K) were found to be positive. In total, the transition between the BARP antigen and the variant surface glycoprotein coats progressively occurs only in premetacyclic cells (Fig. 3I,K), in a comparable way to the bloodstream variant surface glycoprotein replacement by the procyclin coat during the early differentiation in the midgut of the tsetse fly (Roditi et al., 1989).

The age of the centrosome plays a key role in the fate of the progeny during asymmetric divisions in metazoans (Knoblich, 2010). In trypanosomes, there are no centrosomes as such, but a basal body that exhibits an equivalent structure and that is always associated with the flagellum. We reasoned that the two flagella of the 'epitrypo' dividing cells might contain distinct components (Rotureau et al., 2009). To identify candidate markers, we browsed the lists of identified flagellum and basal body proteins (Buisson and Bastin, 2010; Ralston et al., 2009) to search for those likely to display a differential expression between insect and mammalian stages. One such candidate was the calflagin protein family. Calflagins are flagellar calcium-binding proteins known to be present in parasites at the procyclic stage but whose abundance is much higher in bloodstream trypanosomes (Emmer et al., 2010). We first showed that calflagins are also abundant in pre-metacyclic (Fig. 4C,E) and metacyclic (Fig. 4D,E) forms but not in epimastigote parasites (Fig. $4 \mathrm{~A}, \mathrm{E})$. Strikingly, whereas calflagins are present in low and equivalent amounts in both flagella of the 'epi-epi' dividing cells (Fig. 4F- $\mathrm{F}^{\prime}, \mathrm{H}$ ), they are enriched in the new flagellum of the 'epitrypo' dividing cells (Fig. 4G,H), demonstrating their commitment to differentiation. This also suggests that the flagellum is becoming pre-adapted for the infection in the mammalian host. We propose that this asymmetric 'epi-trypo' division represents the first step of differentiation of the epimastigote towards the metacyclic stage.

\section{Dynamics of trypanosome infection in the salivary glands}

The existence of two alternative cell cycles in parasites found in the salivary glands raises the question of their repartition in time and space during an infection. In the present study, salivary gland infections were observed as soon as 14 days after the infective meal. In accordance with recent observations (Oberle et al., 2010; Peacock et al., 2007), the invasion (14 to 21 days after the infective meal) was characterized by a small number of epimastigote parasites sparsely attached in one or both glands (supplementary material Movie 1, part 1; Fig. 5B). After 21 to 28 days, the density of parasites in an infected gland could be so high that the lumen appeared obstructed (supplementary material Movie 1, part 2; Fig. 5C). Both in vivo examination of live parasites in intact or opened salivary glands (supplementary material Movie 1, part 3) and confocal observations of parasites in fixed salivary glands stained with phalloidin (supplementary material Movie 2, Fig. S1) confirmed the presence of dividing cells with the 'epi-epi' and 'epi-trypo' profiles attached to the epithelium. The first freely swimming metacyclic cells were observed from day 18 post-ingestion until the end of the experiments (day 55). To quantify the proportions of each cell type, all the parasites obtained from the salivary glands of two groups of flies dissected at early ( $<21$ days) or late $(>28$ days) stages of infection (Fig. 5A) were characterized by DAPI staining. The efficiency of the parasite extraction from the glands was first assessed with GFPexpressing trypanosomes. In total, $1 \mathrm{~K} 1 \mathrm{~N}$ cells (epimastigotes and trypomastigotes) were found to account for $57 \%$ (early infection) and $73 \%$ (late infection) of the cells (Fig. 5A). In early infections, there was a predominance of parasites in the 'epi-epi' configuration $(2 \mathrm{~K} 1 \mathrm{~N}$ and $2 \mathrm{~K} 2 \mathrm{~N}$ ) that represented $75 \%$ of all the dividing cells. By sharp contrast, the balance was tipped over at later stages of infection with a predominance $(62 \%)$ of the 'epi-trypo' profile.

\section{DISCUSSION}

Taken together, these results demonstrate the existence of two alternative proliferation patterns occurring simultaneously in the salivary glands of the tsetse fly (Fig. 6; supplementary material Table $\mathrm{S} 1)$. At the early stage of infection, the 'epi-epi' cell division is predominant, presumably to allow rapid amplification and establishment of the infection by colonization of the salivary glands. 


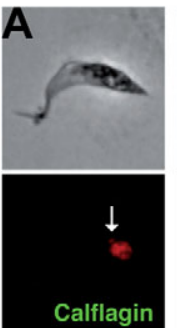

Epimastigote

E
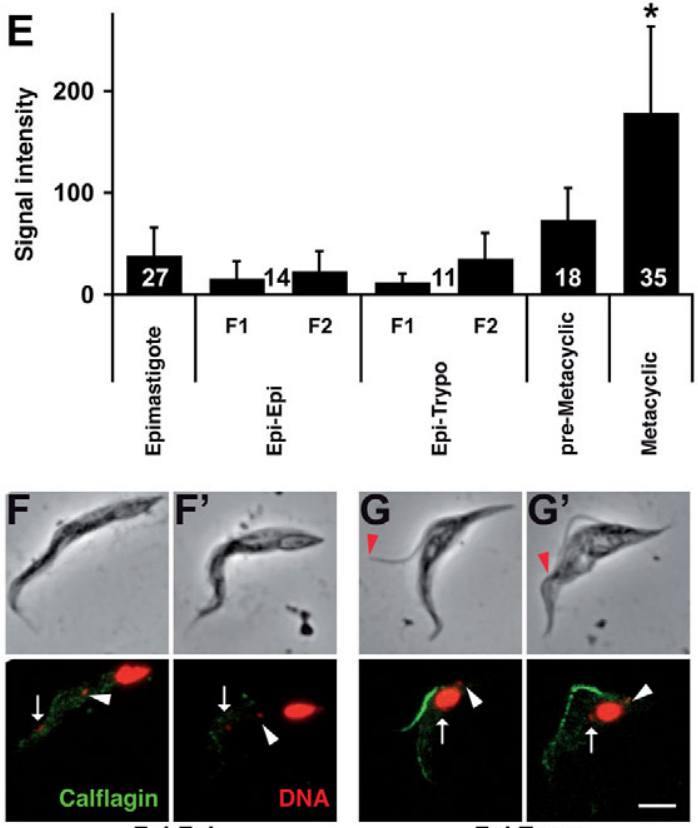

Epi-Epi
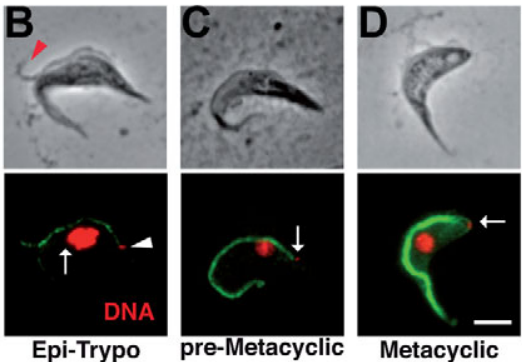

Metacyclic
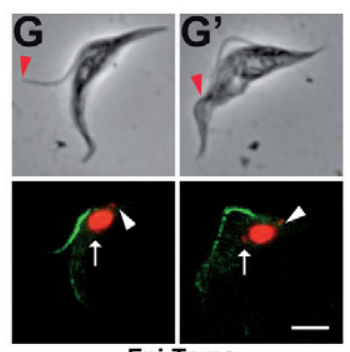

Epi-Trypo

\begin{tabular}{lccc}
\hline F2 / F1 & Epi-Epi $(n=14)$ & Epi-Trypo $(n=11)$ & $p$ \\
\hline Mean & $1.3 \pm 0.3$ & $2.0 \pm 0.4$ & 0.00002 \\
Maximum & $1.2 \pm 0.2$ & $2.2 \pm 0.5$ & 0.00001 \\
\hline
\end{tabular}

Fig. 4. Calflagins as early molecular markers for differentiation. $(\mathbf{A}-\mathbf{D}, \mathbf{F}, \mathbf{G})$ Trypanosomes from salivary glands were fixed in methanol for 30 seconds and stained with 4',6-diamidino-2-phenylindole (DNA in red) and anti-calflagins (calflagins in green). Epimastigotes (A), 'epi-epi' cells $\left(F, F^{\prime}\right)$, 'epi-trypo' cells $(B, G, G$ '), pre-metacyclic cells $(C)$ and metacyclic trypomastigotes $(D)$ were identified according to the number and relative positioning of their kinetoplasts and nuclei. The positions of the old (white arrow) and new (white arrowhead) kinetoplasts are indicated, as well as the tip of the new flagellum (red arrowhead) when present. Scale bars: $5 \mu \mathrm{m}$. (E) Mean normalized flagellum fluorescence intensity \pm s.d. (in arbitrary units) obtained upon staining calflagins in the indicated trypanosome stages from salivary glands. $n$ is given inside columns; ${ }^{*} P<0.001$, two-tailed unpaired Student's $t$-test, when compared with all other groups. (H) Comparison of the ratios between calflagin staining intensities measured in the new flagellum (F2) and the old flagellum (F1) of the same dividing cell according to the division type. Ratios \pm s.d. of the mean and maximum values of normalized fluorescence measured along each entire flagellum. $P$ from two-tailed unpaired Student's $t$-test.

This is taking place in a 'mosaic' pattern as reported previously (Peacock et al., 2007), with small colonies of parasites spreading at different locations in the epithelium that would ultimately grow to join each other (supplementary material Movie 1, Fig. S1A; Fig. 5B,C). Some 'epi-trypo' asymmetric divisions are also detected, leading to early production of metacyclic parasites. The induction of
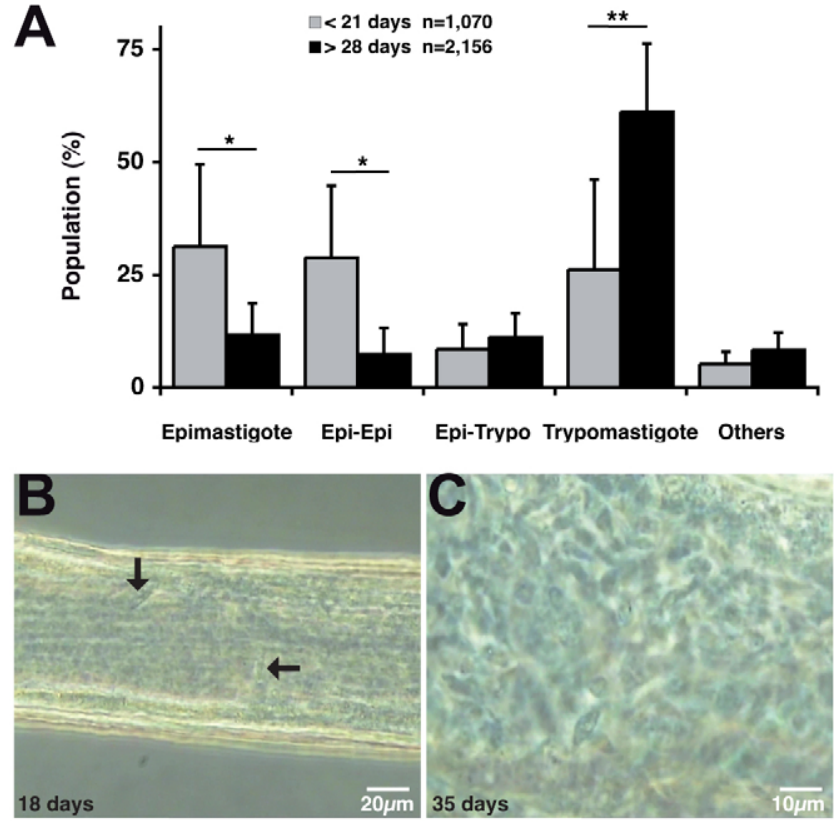

Fig. 5. Parasite population dynamics during the course of the salivary gland infection. (A) Parasite populations in the salivary glands according to the time of infection. Mean percentages \pm s.d. of cells in each stage out of the total number of parasites in the salivary glands from flies dissected less than 21 days (early infection in gray, $n=1070$ cells from six flies) or more than 28 days (late infection in black, $n=2156$ cells from six flies) identified by DNA staining with $4^{\prime}, 6$ diamidino-2-phenylindole. 'Epi-epi' and 'epi-trypo' groups include $2 \mathrm{~K} 1 \mathrm{~N}$ and $2 \mathrm{~K} 2 \mathrm{~N}$ cells. 'Others' includes cells with $\mathrm{nKnN}$ (with $n>2$ ), zoids $(1 \mathrm{KON})$ and few dividing trypomastigotes. ${ }^{*} P<0.01,{ }^{*} P<0.05$, two-tailed unpaired Student's t-test. (B) Still image from supplementary material Movie 1 (part 1) showing the early infection (18 days after ingestion) of a salivary gland by epimastigote parasites attached to the epithelium (black arrows). Scale bar: $20 \mu \mathrm{m}$. (C) Still image from supplementary material Movie 1 (part 2) showing the presence of numerous parasites in the lumen of a salivary gland at a later step of infection (21 days after ingestion). Scale bar: $10 \mu \mathrm{m}$.

epi-trypo differentiation could be triggered by physical contact between attached epimastigotes. Alternatively, the emergence of 'epitrypo' cells could be associated with the locally high density of attached parasites. Indeed, the high concentration of cells on a restricted surface could lead to the local production of trypanosome molecules that, in turn, would result in a local quorum-sensing-like phenomenon similar to what has been proposed for the production of stumpy cells during bloodstream infection (Vassella et al., 1997). In contrast to epimastigotes that are attached to the epithelium, metacyclic parasites are released in the saliva. During each bloodmeal, most of them are ejected with the saliva. This drastic change in the environment could also activate the entry of some attached epimastigotes in the 'epi-trypo' cycle to ensure the rapid production of a new pool of infective cells.

In both types of cell division, the tip of the new flagellum beats freely when cells are close to cytokinesis. This movement could be necessary to allow cell separation, as observed during division of procyclic trypanosomes, where inhibition of motility prevents the last step of cytokinesis in vitro. Interestingly, this phenomenon can be relieved upon mechanical shaking of the culture (Branche et al., 2006; Ralston et al., 2006). As we did not observe any living dividing cells attached with their two flagella, the future daughter 


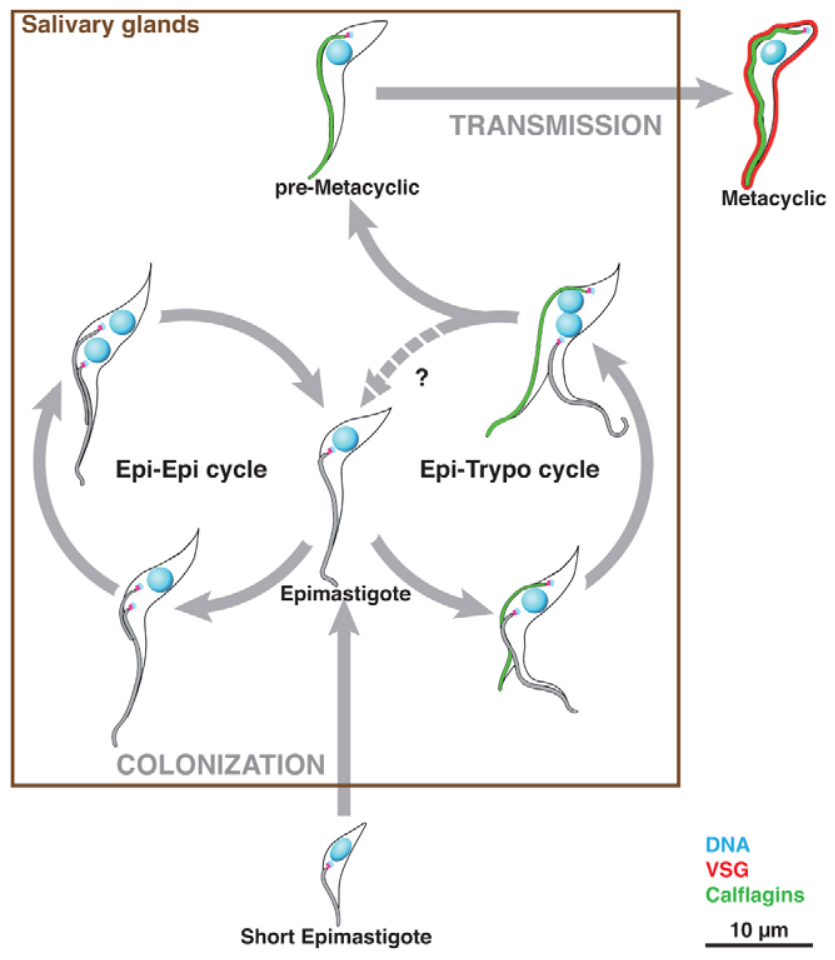

Fig. 6. Contribution of the two modes of proliferation in the balance between colonization and transmission. Schematics of the proposed parasite development in the salivary glands. Stages inside the box are found attached to the salivary gland epithelium. Basal bodies in pink, calflagins in green and variant surface glycoprotein (VSG) coat in red.

cell is likely to be released in the lumen and to attach rapidly to the epithelium again, either to initiate a novel round of cell division or to allow the completion of the differentiation into the metacyclic form. In the case of the 'epi-trypo' division, the fate of the epimastigote progeny remains to be determined. The resulting attached epimastigote could re-iterate that process; hence, behaving like a 'stem cell' that ensures the maintenance of the attached population at the surface of the epithelium and the continuous production of pre-metacyclic cells. Alternatively, this epimastigote could die after the asymmetric division, a situation that has been proposed to occur for the long epimastigote cell resulting from another asymmetric division occurring in the proventriculus and foregut earlier in the parasite cycle (Sharma et al., 2009; Van Den Abbeele et al., 1999). In that case, the coupling of the asymmetric division to the 'epi-epi' cycle would be essential to maintain the infection in the salivary glands. It is also noteworthy that some epimastigotes were recently observed to achieve the first meiotic division $(<20 \%$ with a $T$. $b$. brucei $\mathrm{J} 10$ background) (Peacock et al., 2011). This observation is compatible with the model proposed here, as these parasites could correspond to a part of the 'epi-epi' dividing cell population. However, the frequency of meiotic events in our conditions ( $T$. b. brucei AnTat1.1 in $G$. $m$. morsitans from Zimbabwe) remains undetermined. The second step of the meiotic program and the subsequent cell fusion have now to be elucidated and compared between African trypanosome species and strains.

Our data do not definitely exclude the possibility of a direct differentiation of an attached epimastigote into a pre-metacyclic trypomastigote as suggested by Vickerman (Vickerman, 1985).
Assessing the relative contributions of the two hypotheses in the metacyclogenetic process appears to be technically challenging at that time. First, live cell time-lapse imaging is technically not feasible in vivo in the fly yet. Second, to our knowledge, conditions for the culture of salivary gland parasites that reproduce trypanosome differentiation have never been reported. Thus, the monitoring of the kinetoplast movements in a single cell attached to the salivary gland epithelium of a living tsetse fly or to an artificial substratum appears difficult to obtain. However, it is noteworthy that no argument for a direct differentiation has been found in the present study.

Metacyclic parasites are pre-adapted to life in the mammalian host and possess the variant surface glycoprotein coat that is not yet detected during the 'epi-trypo' division, at least by using the anti-CRD indirect probe. The BARP antigen coat is replaced by the variant surface glycoprotein coat after cell division, exactly as observed for the loss of the variant surface glycoproteins and the emergence of procyclin when bloodstream parasites differentiate into procyclic cells (Roditi et al., 1989). By contrast, the flagellum is assembled only once during the cell cycle, a process that takes place before cytokinesis. The higher amounts of calflagins in the new flagellum compared with the old flagellum of the 'epi-trypo' cell could constitute the first step of a pre-adaptation to life in the mammalian host, where calflagins are highly abundant and contribute to infectivity (Emmer et al., 2010). This reveals preferential targeting of membrane proteins to this flagellum versus the old one.

The progression of $T$. brucei infection in a tsetse fly is a slow and complex process. Even in countries where the disease is endemic, fewer than $0.1 \%$ of flies carry metacyclic parasites (Aksoy et al., 2003; Brun et al., 2009). Our results reveal how the parasite has evolved to compensate these low infection rates, by developing cellular processes remarkably adapted to the high life span of tsetse flies (1 to 9 months) (Aksoy et al., 2003). The balance between the two patterns of cell cycle in parasites attached to tsetse salivary glands (Fig. 6) would ensure continuous production of numerous infective cells for long periods, allowing the efficient diffusion of African trypanosomes over time and space. This strategy greatly contrasts with those evolved by Plasmodium in Anopheles mosquitoes or by Leishmania in sandflies, which are transmitted by more abundant vectors with a much shorter life span (Aly et al., 2009; Bates, 2007; Cohuet et al., 2010). Overall, the new mode of asymmetric division discovered in the salivary glands explains the continuous production of infective metacyclic parasites, turning the tsetse flies into efficient and long-lasting vectors for African trypanosomes.

\section{Acknowledgements}

We thank the 'TRYPANOSOM'-UMR 177 IRD CIRAD team (Campus International de Baillarguet, Montpellier, France) headed by G. Cuny for generously providing tsetse flies, especially B. Tchicaya and J. Janelle. We are grateful to J. Van Den Abbeele for providing the trypanosome cell line. We acknowledge I. Roditi, W. Gibson, J. Bangs, D. Engman, D. Robinson, M. Carrington and K. Gull for providing various antibodies and/or plasmids. We thank G. Milon, L. Kohl, L. Vincensini, K. Vickerman, F. Cox, L. Tetley, A. Scherf, G. Spaeth, F. Bringaud and D. Robinson for critical reading of the manuscript.

\section{Funding}

This work was funded by the Centre National de la Recherche Scientifique (CNRS), the Institut Pasteur and by an L'Agence nationale de la recherche Maladies infectieuses et leur environnement (ANR-MIE) grant [ANR-08-MIE027]. B.R. was funded by a Roux post-doctoral fellowship, I.S. by a Fonds National de la Recherche (FNR) fellowship and J.B. by a Bourse du Ministère de I'Enseignement et de la Recherche, Ecole Doctorale interdisciplinaire du Vivant [ED327]. 


\section{Competing interests statement}

The authors declare no competing financial interests.

\section{Author contributions}

B.R. carried out all the experiments and wrote the manuscript. I.S. participated in fly maintenance, in the immunofluorescence analysis with the anti-BARP and prepared Movie 3. J.B. drew the schematics. I.S. and P.B. discussed the results and commented on the manuscript.

\section{Supplementary material}

Supplementary material available online at

http://dev.biologists.org/lookup/suppl/doi:10.1242/dev.072611/-/DC1

\section{References}

Aksoy, S., Gibson, W. C. and Lehane, M. J. (2003). Interactions between tsetse and trypanosomes with implications for the control of trypanosomiasis. Adv. Parasitol. 53, 1-83.

Aly, A. S., Vaughan, A. M. and Kappe, S. H. (2009). Malaria parasite development in the mosquito and infection of the mammalian host. Annu. Rev. Microbiol. 63, 195-221.

Bates, P. A. (2007). Transmission of Leishmania metacyclic promastigotes by phlebotomine sand flies. Int. J. Parasitol. 37, 1097-1106.

Berriman, M., Ghedin, E., Hertz-Fowler, C., Blandin, G., Renauld, H. Bartholomeu, D. C., Lennard, N. J., Caler, E., Hamlin, N. E., Haas, B. et al. (2005). The genome of the African trypanosome Trypanosoma brucei. Science 309, 416-422.

Bingle, L. E., Eastlake, J. L., Bailey, M. and Gibson, W. C. (2001). A novel GFP approach for the analysis of genetic exchange in trypanosomes allowing the in situ detection of mating events. Microbiology 147, 3231-3240.

Birkett, C. R., Foster, K. E., Johnson, L. and Gull, K. (1985). Use of monoclona antibodies to analyse the expression of a multi-tubulin family. FEBS Lett. 187 $211-218$.

Bonhivers, M., Landrein, N., Decossas, M. and Robinson, D. R. (2008). A monoclonal antibody marker for the exclusion-zone filaments of Trypanosoma brucei. Parasit. Vectors 1, 21

Branche, C., Kohl, L., Toutirais, G., Buisson, J., Cosson, J. and Bastin, P. (2006). Conserved and specific functions of axoneme components in trypanosome motility. J. Cell Sci. 119, 3443-3455.

Brun, R., Blum, J., Chappuis, F. and Burri, C. (2009). Human African trypanosomiasis. Lancet 375, 148-159.

Buisson, J. and Bastin, P. (2010). Flagellum Structure and Function in Trypanosomes. In Structures and Organelles in Pathogenic Protists (ed. W. De Souza), pp 63-86. Berlin, Heidelberg: Springer-Verlag.

Cardoso de Almeida, M. L. and Turner, M. J. (1983). The membrane form of variant surface glycoproteins of Trypanosoma brucei. Nature 302, 349-352.

Chappuis, F., Lima, M. A., Flevaud, L. and Ritmeijer, K. (2010). Human African trypanosomiasis in areas without surveillance. Emerg. Infect. Dis. 16, 354-356.

Cohuet, A., Harris, C., Robert, V. and Fontenille, D. (2010). Evolutionary forces on Anopheles: what makes a malaria vector? Trends Parasitol. 26, 130-136.

Cross, G. A. (1975). Identification, purification and properties of clone-specific glycoprotein antigens constituting the surface coat of Trypanosoma brucei. Parasitology 71, 393-417.

Emmer, B. T., Daniels, M. D., Taylor, J. M., Epting, C. L. and Engman, D. M. (2010). Calflagin inhibition prolongs host survival and suppresses parasitemia in Trypanosoma brucei infection. Eukaryot. Cell 9, 934-942.

Fevre, E. M., Odiit, M., Coleman, P. G., Woolhouse, M. E. and Welburn, S. C. (2008). Estimating the burden of rhodesiense sleeping sickness during an outbreak in Serere, eastern Uganda. BMC Public Health 8, 96.

Giroud, C., Ottones, F., Coustou, V., Dacheux, D., Biteau, N., Miezan, B., Van Reet, N., Carrington, M., Doua, F. and Baltz, T. (2009). Murine Models for Trypanosoma brucei gambiense disease progression-from silent to chronic infections and early brain tropism. PLoS Negl. Trop. Dis. 3, e509.

Grab, D. J., Webster, P. and Verjee, Y. (1984). The intracellular pathway and assembly of newly formed variable surface glycoprotein of Trypanosoma brucei. Proc. Natl. Acad. Sci. USA 81, 7703-7707.

Kilmartin, J. V., Wright, B. and Milstein, C. (1982). Rat monoclonal antitubulin antibodies derived by using a new nonsecreting rat cell line. J. Cell Biol. 93, 576582.

Knoblich, J. A. (2010). Asymmetric cell division: recent developments and their implications for tumour biology. Nat. Rev. Mol. Cell Biol. 11, 849-860.

Kohl, L., Sherwin, T. and Gull, K. (1999). Assembly of the paraflagellar rod and the flagellum attachment zone compex during the Trypanosoma brucei cell cycle. J. Eukaryot. Microbiol. 46, 105-109.

Le Ray, D., Barry, J. D., Easton, C. and Vickerman, K. (1977). First tsetse fly transmission of the "AnTat" serodeme of Trypanosoma brucei. Ann. Soc. Belg. Med. Trop. 57, 369-381.

Oberle, M., Balmer, O., Brun, R. and Roditi, I. (2010). Bottlenecks and the maintenance of minor genotypes during the life cycle of Trypanosoma brucei. PLoS Pathog. 6, e1001023.
Otieno, L. H. and Darji, N. (1979). The abundance of pathogenic African trypanosomes in the salivary secretions of wild Glossina pallidipes. Ann. Trop. Med. Parasitol. 73, 583-588

Peacock, L., Ferris, V., Bailey, M. and Gibson, W. (2007). Dynamics of infection and competition between two strains of Trypanosoma brucei brucei in the tsetse fly observed using fluorescent markers. Kinetoplastid Biol. Dis. 6, 4

Peacock, L., Ferris, V., Sharma, R., Sunter, J., Bailey, M., Carrington, M. and Gibson, W. (2011). Identification of the meiotic life cycle stage of Trypanosoma brucei in the tsetse fly. Proc. Natl. Acad. Sci. USA 108, 3671-3676.

Pradel, L. C., Bonhivers, M., Landrein, N. and Robinson, D. R. (2006). NIMArelated kinase TbNRKC is involved in basal body separation in Trypanosoma brucei. J. Cell Sci. 119, 1852-1863.

Ralston, K. S., Lerner, A. G., Diener, D. R. and Hill, K. L. (2006). Flagellar motility contributes to cytokinesis in Trypanosoma brucei and is modulated by an evolutionarily conserved dynein regulatory system. Eukaryot. Cell 5, 696-711.

Ralston, K. S., Kabututu, Z. P., Melehani, J. H., Oberholzer, M. and Hill, K. L. (2009). The Trypanosoma brucei flagellum: moving parasites in new directions. Annu. Rev. Microbiol. 63, 335-362.

Robinson, D. R. and Gull, K. (1991). Basal body movements as a mechanism fo mitochondrial genome segregation in the trypanosome cell cycle. Nature $\mathbf{3 5 2}$, 731-733.

Roditi, I., Schwarz, H., Pearson, T. W., Beecroft, R. P., Liu, M. K., Richardson, J. P., Buhring, H. J., Pleiss, J., Bulow, R., Williams, R. O. et al. (1989). Procyclin gene expression and loss of the variant surface glycoprotein during differentiation of Trypanosoma brucei. J. Cell Biol. 108, 737-746.

Rotureau, B., Morales, M. A., Bastin, P. and Spath, G. F. (2009). The flagellumMAP kinase connection in Trypanosomatids: a key sensory role in parasite signaling and development? Cell Microbiol. 11, 710-718.

Rotureau, B. Subota, I. and Bastin, P. (2011). Molecular bases of cytoskeleton plasticity during the Trypanosoma brucei parasite cycle. Cell Microbiol. 13, 705 716.

Sharma, R., Peacock, L., Gluenz, E., Gull, K., Gibson, W. and Carrington, M. (2008). Asymmetric cell division as a route to reduction in cell length and change in cell morphology in trypanosomes. Protist 159, 137-151.

Sharma, R., Gluenz, E., Peacock, L., Gibson, W., Gull, K. and Carrington, M (2009). The heart of darkness: growth and form of Trypanosoma brucei in the tsetse fly. Trends Parasitol. 25, 517-524.

Sherwin, T. and Gull, K. (1989). Visualization of detyrosination along single microtubules reveals novel mechanisms of assembly during cytoskeletal duplication in trypanosomes. Cell 57, 211-221.

Simarro, P. P., Jannin, J. and Cattand, P. (2008). Eliminating human African trypanosomiasis: where do we stand and what comes next? PLoS Med. 5, e55.

Steiger, R. F. (1973). On the ultrastructure of Trypanosoma (Trypanozoon) bruce in the course of its life cycle and some related aspects. Acta Trop. 30, 64-168.

Taylor, J. E. and Rudenko, G. (2006). Switching trypanosome coats: what's in the wardrobe? Trends Genet. 22, 614-620.

Tetley, L. and Vickerman, K. (1985). Differentiation in Trypanosoma brucei: hostparasite cell junctions and their persistence during acquisition of the variable antigen coat. J. Cell Sci. 74, 1-19.

Tetley, L., Turner, C. M., Barry, J. D., Crowe, J. S. and Vickerman, K. (1987) Onset of expression of the variant surface glycoproteins of Trypanosoma bruce in the tsetse fly studied using immunoelectron microscopy. J. Cell Sci. 87, 363372 .

Urwyler, S., Studer, E., Renggli, C. K. and Roditi, I. (2007). A family of stagespecific alanine-rich proteins on the surface of epimastigote forms of Trypanosoma brucei. Mol. Microbiol. 63, 218-228.

Van Den Abbeele, J., Claes, Y., van Bockstaele, D., Le Ray, D. and Coosemans, M. (1999). Trypanosoma brucei spp. development in the tsetse fly: characterization of the post-mesocyclic stages in the foregut and proboscis Parasitology 118, 469-478.

Van Den Abbeele, J., Caljon, G., De Ridder, K., De Baetselier, P. and Coosemans, M. (2010). Trypanosoma brucei modifies the tsetse salivary composition, altering the fly feeding behavior that favors parasite transmission PLoS Pathog. 6, e1000926.

Vassella, E., Reuner, B., Yutzy, B. and Boshart, M. (1997). Differentiation of African trypanosomes is controlled by a density sensing mechanism which signals cell cycle arrest via the CAMP pathway. J. Cell Sci. 110, 2661-2671.

Vassella, E., Oberle, M., Urwyler, S., Renggli, C. K., Studer, E., Hemphill, A. Fragoso, C., Butikofer, P., Brun, R. and Roditi, I. (2009). Major surface glycoproteins of insect forms of Trypanosoma brucei are not essential for cyclical transmission by tsetse. PLOS ONE 4, e4493.

Vickerman, K. (1978). Antigenic variation in trypanosomes. Nature 273, 613-617.

Vickerman, K. (1985). Developmental cycles and biology of pathogenic trypanosomes. Br. Med. Bull. 41, 105-114.

Vickerman, K., Tetley, L., Hendry, K. A. and Turner, C. M. (1988). Biology of African trypanosomes in the tsetse fly. Biol. Cell 64, 109-119.

Zamze, S. E., Ferguson, M. A., Collins, R., Dwek, R. A. and Rademacher, T. W. (1988). Characterization of the cross-reacting determinant (CRD) of the glycosylphosphatidylinositol membrane anchor of Trypanosoma brucei variant surface glycoprotein. Eur. J. Biochem. 176, 527-534. 